\title{
CORRECTION
}

\section{Correction to: Bacteria: back pain, leg pain and Modic sign-a surgical multicenter comparative study}

Peter Fritzell ${ }^{1}$. Christina Welinder-Olsson ${ }^{2}$ - Bodil Jönsson ${ }^{2} \cdot$ Åsa Melhus $^{3} \cdot$ Siv G. E. Andersson ${ }^{4}$. Tomas Bergström ${ }^{5} \cdot$ Hans Tropp $^{6} \cdot$ Paul Gerdhem $^{7}$. Olle Hägg ${ }^{8} \cdot$ Hans Laestander $^{8}$ - Björn Knutsson ${ }^{9}$. Anders Lundin $^{10} \cdot$ Per Ekman $^{11}$. Eric Rydman ${ }^{11} \cdot$ Mikael Skorpil $^{12}$

Published online: 29 October 2019

(c) Springer-Verlag GmbH Germany, part of Springer Nature 2019

Correction to: European Spine Journal https://doi.org/10.1007/s00586-019-06164-1

Unfortunately, the 5th author name was incorrectly published in the original paper. The complete correct name is given below.

Siv G. E. Andersson.

The original article has been updated.

Publisher's Note Springer Nature remains neutral with regard to jurisdictional claims in published maps and institutional affiliations.

The original article can be found online at https://doi.org/10.1007/ s00586-019-06164-1.

Peter Fritzell

peterfritzell@gmail.com

Extended author information available on the last page of the article 


\section{Affiliations}

\section{Peter Fritzell ${ }^{1}$ (1) . Christina Welinder-Olsson ${ }^{2} \cdot$ Bodil Jönsson $^{2} \cdot$ Åsa Melhus ${ }^{3} \cdot$ Siv G. E. Andersson ${ }^{4}$. Tomas Bergström ${ }^{5} \cdot$ Hans Tropp $^{6} \cdot$ Paul Gerdhem $^{7}$. Olle Hägg ${ }^{8} \cdot$ Hans Laestander $^{8}$ • Björn Knutsson ${ }^{9}$. Anders Lundin ${ }^{10} \cdot$ Per Ekman $^{11}$. Eric Rydman ${ }^{11}$. Mikael Skorpil ${ }^{12}$}

Christina Welinder-Olsson

christina.welinder-olsson@ vgregion.se

Bodil Jönsson

bodil.jonsson@vgregion.se

Åsa Melhus

asa.melhus@medsci.uu.se

Siv G. E. Andersson

siv.andersson@icm.uu.se

Tomas Bergström

tomas.bergstrom@microbio.gu.se

Hans Tropp

hans.tropp@ regionostergotland.se

Paul Gerdhem

paul.gerdhem@sll.se

Olle Hägg

olle.hagg@ spinecenter.se

Hans Laestander

hans.laestander@spinecenter.se

Björn Knutsson

bjornknutsson4@gmail.com

Anders Lundin

alundin54@gmail.com

Per Ekman

per.a.ekman@sll.se
Eric Rydman

eric.rydman@sll.se

Mikael Skorpil

mikael.skorpil@ki.se

1 Futurum Academy, Länssjukhuset Ryhov, 55185 Jönköping, Sweden

2 Sahlgrenska University Hospital, 41346 Göteborg, Sweden

3 Uppsala University Hospital, 75185 Uppsala, Sweden

4 Department of Cell and Molecular Biology, Biomedical Center, Uppsala University, 75123 Uppsala, Sweden

5 Department of Infectious Diseases, Institute of Biomedicine, University of Gothenburg, 41346 Göteborg, Sweden

6 Linköping University, 58183 Linköping, Sweden

7 Karolinska University Hospital, 14186 Stockholm, Sweden

8 Spine Center Göteborg, 42130 Västra Frölunda, Sweden

9 Sundsvall Hospital, 85786 Sundsvall, Sweden

10 Örebro University Hospital, 70185 Örebro, Sweden

11 Södersjukhuset, 11883 Stockholm, Sweden

12 Karolinska University Hospital, 17164 Stockholm, Sweden 